\title{
Combinatorial Gelfand Models
}

\author{
Ron M. Adin ${ }^{1}$ and Alex Postnikov ${ }^{2}$ and Yuval Roichman ${ }^{3}$ \\ ${ }^{1}$ Department of Mathematics, Bar-Ilan University, Ramat-Gan 52900, Israel radin@math.biu.ac.i I \\ ${ }^{2}$ Dept. of Applied Mathematics, Massachusetts Institute of Technology, MA 02139, USA apost@math.mit.edu \\ ${ }^{3}$ Department of Mathematics, Bar-Ilan University, Ramat-Gan 52900, Israel yuvalr@math.biu.ac.i I
}

\begin{abstract}
A combinatorial construction of Gelfand models for the symmetric group, for its Iwahori-Hecke algebra and for the hyperoctahedral group is presented.
\end{abstract}

Keywords: symmetric group, hyperoctahedral group, Iwahori-Hecke algebra, descents, inversions, character formulas, Gelfand model

\section{Introduction}

A complex representation of a group or an algebra $A$ is called a Gelfand model for $A$, or simply a model, if it is equivalent to the multiplicity free direct sum of all $A$-irreducible representations.

Models (for compact Lie groups) were first constructed by Bernstein, Gelfand and Gelfand [8]. Constructions of models for the symmetric group, using induced representations from centralizers, were found by Klyachko [13, 14] and by Inglis, Richardson and Saxl [11]; see also [6, 21, 4, 3, 5]. Our goal is to determine an explicit and simple combinatorial action which gives a model for the symmetric group and its Iwahori-Hecke algebra and for the hyperoctahedral group. The descent set of a permutation plays a crucial role in the construction and in a resulting character formula.

The rest of the paper is organized as follows. Main results are given in Sections 2-4. Proofs are sketched in Sections 5-7. Section 8 concludes with remarks and open problems.

This is an extended abstract. For a more detailed version see [1].

\section{The Symmetric Group}

Let $S_{n}$ be the symmetric group on $n$ letters, $S=\left\{s_{1}, \ldots, s_{n-1}\right\}$ the set of simple reflections in $S_{n}$, $I_{n}=\left\{\pi \in S_{n} \mid \pi^{2}=i d\right\}$ its the set of involutions, and $V_{n}:=\operatorname{span}_{\mathbb{Q}}\left\{C_{w} \mid w \in I_{n}\right\}$ a vector space over $\mathbb{Q}$ formally spanned by the involutions.

Recall the standard length function on the symmetric group

$$
\ell(\pi):=\min \left\{\ell \mid \pi=s_{i_{1}} s_{i_{2}} \cdots s_{i_{\ell}}, s_{i_{j}} \in S(\forall j)\right\},
$$

the descent set

$$
\operatorname{Des}(\pi):=\{s \in S \mid \ell(\pi s)<\ell(\pi)\},
$$

1365-8050 (c) 2008 Discrete Mathematics and Theoretical Computer Science (DMTCS), Nancy, France 
and the descent number $\operatorname{des}(\pi):=\# \operatorname{Des}(\pi)$.

Define a map $\rho: S \rightarrow G L\left(V_{n}\right)$ by

$$
\rho(s) C_{w}:=\operatorname{sign}(s ; w) \cdot C_{s w s} \quad\left(\forall s \in S, w \in I_{n}\right)
$$

where

$$
\operatorname{sign}(s ; w):= \begin{cases}-1, & \text { if } s w s=w \text { and } s \in \operatorname{Des}(w) \\ 1, & \text { otherwise. }\end{cases}
$$

Theorem 2.1 determines an $S_{n}$-representation.

Theorem 2.2 $\rho$ determines a Gelfand model for $S_{n}$.

\section{Hecke Algebra Action}

Consider $H_{n}(q)$, the Hecke algebra of the symmetric group $S_{n}$ (say over the field $\mathbb{Q}\left(q^{1 / 2}\right)$ ), generated by $\left\{T_{i} \mid 1 \leq i<n\right\}$ with the defining relations

$$
\begin{gathered}
\left(T_{i}+q\right)\left(T_{i}-1\right)=0 \quad(\forall i), \\
T_{i} T_{j}=T_{j} T_{i} \quad \text { if } \quad|i-j|>1, \\
T_{i} T_{i+1} T_{i}=T_{i+1} T_{i} T_{i+1} \quad(1 \leq i<n-1) .
\end{gathered}
$$

Note that some authors use a slightly different notation, with $T_{i}$ consistently replaced by $-T_{i}$. The current definition gives a more convenient description of the characters, see Proposition 3.3 below.

In order to construct an extended signed conjugation, which gives a model for $H_{n}(q)$, we extend the standard notions of length and weak order. Recall that the (left) weak order $\leq_{L}$ on $S_{n}$ is the reflexive and transitive closure of the relation: $w \prec_{L} w s$ if $s \in S$ and $\ell(w)+1=\ell(s w)$.

Definition 3.1 Define the involutive length of an involution $w \in I_{n}$ with cycle type $\left(1^{n-2 k} 2^{k}\right)$ as

$$
\hat{\ell}(w):=\min \left\{\ell(v) \mid w=v s_{1} s_{3} \cdots s_{2 k-1} v^{-1}\right\}
$$

where $\ell(v)$ is the standard length of $v \in S_{n}$.

Define the involutive weak order $\leq_{I}$ on $I_{n}$ as the reflexive and transitive closure of the relation: $w \prec_{I}$ sws if $s \in S$ and $\hat{\ell}(w)+1=\hat{\ell}(s w s)$.

Define a map $\rho_{q}: S \rightarrow G L\left(V_{n}\right)$ by

$$
\rho_{q}\left(T_{s}\right) C_{w}:= \begin{cases}-q C_{w}, & \text { if } s w s=w \text { and } s \in \operatorname{Des}(w) ; \\ C_{w}, & \text { if } s w s=w \text { and } s \notin \operatorname{Des}(w) ; \\ (1-q) C_{w}+q C_{s w s}, & \text { if } w \prec_{I} s w s ; \\ C_{s w s}, & \text { if } s w s \prec_{I} w .\end{cases}
$$

Theorem 3.2 $\rho_{q}$ is a Gelfand model for $H_{n}(q)$ ( $q$ indeterminate); namely, 
(1) $\rho_{q}$ is an $H_{n}(q)$-representation.

(2) $\rho_{q}$ is equivalent to the multiplicity free sum of all irreducible $H_{n}(q)$-representations.

The proof involves Lusztig's version of Tits' deformation theorem [17]. For other versions of this theorem see $[9, \S 4],[10, \S 68 . \mathrm{A}]$ and [7].

Let $\mu=\left(\mu_{1}, \mu_{2}, \ldots, \mu_{t}\right)$ be a partition of $n$ and let $a_{j}:=\sum_{i=1}^{j} \mu_{i}(0 \leq j \leq t)$. A permutation $\pi \in S_{n}$ is $\mu$-unimodal if for every $0 \leq j<t$ there exists $1 \leq d_{j} \leq \mu_{j+1}$ such that

$$
\pi_{a_{j}+1}<\pi_{a_{j}+2}<\cdots<\pi_{a_{j}+d_{j}}>\pi_{a_{j}+d_{j}+1}>\pi_{a_{j}+d_{j}+2}>\cdots>\pi_{a_{j+1}} .
$$

The character of $\rho_{q}$ may be expressed as a generating function for the descent number over $\mu$-unimodal involutions.

Proposition 3.3

$$
\operatorname{Tr}\left(\rho_{q}\left(T_{\mu}\right)\right)=\sum_{\left\{w \in I_{n} \mid w \text { is } \mu \text {-unimodal }\right\}}(-q)^{\operatorname{des}(w)}
$$

where $T_{\mu}:=T_{1} T_{2} \cdots T_{\mu_{1}-1} T_{\mu_{1}+1} \cdots T_{\mu_{1}+\ldots+\mu_{t}-1}$ is the subproduct of $T_{1} T_{2} \cdots T_{n-1}$ omitting the factors $T_{\mu_{1}+\cdots+\mu_{i}}$ for all $1 \leq i<t$.

\section{The Hyperoctahedral Group}

Let $B_{n}$ be the Weyl group of type $B, S^{B}$ - the set of simple reflections in $B_{n}, I_{n}^{B}$ - the set of involutions in $B_{n}$, and $V_{n}^{B}:=\operatorname{span}_{\mathbb{Q}}\left\{C_{w} \mid w \in I_{n}^{B}\right\}$ a vector space over $\mathbb{Q}$ spanned by the involutions. Recall that $B_{n}=\mathbb{Z}_{2}\left\langle S_{n}\right.$, so that each element $w \in B_{n}$ is identified with a pair $(v, \sigma)$, where $v \in \mathbb{Z}_{2}^{n}$ and $\sigma \in S_{n}$. Denote $|w|:=\sigma$.

Define a map $\rho^{B}: S^{B} \rightarrow G L\left(V_{n}\right)$ by

$$
\rho^{B}(s) C_{w}:=\operatorname{sign}(s ; w) \cdot C_{s w s} \quad\left(\forall s \in S^{B}, w \in I_{n}^{B}\right)
$$

where, for $s=s_{0}=((1,0, \ldots, 0), i d)$, the exceptional Coxeter generator, the sign is

$$
\operatorname{sign}\left(s_{0} ; w\right):= \begin{cases}-1, & \text { if } s w s=w \text { and } s_{0} \in \operatorname{Des}(w) \\ 1, & \text { otherwise }\end{cases}
$$

and for $s \neq s_{0}$ the sign is

$$
\operatorname{sign}(s ; w):= \begin{cases}-1, & \text { if } s w s=w \text { and } s \in \operatorname{Des}(|w|) \\ 1 & \text { otherwise }\end{cases}
$$

Theorem 4.1 $\rho^{B}$ is a Gelfand model for $B_{n}$.

For a proof and generalizations see [2]. 


\section{Characters}

\subsection{Character Formula}

The following classical result follows from the work of Frobenius and Schur, see [12, §4] and [23, §7, Ex. 69].

Theorem 5.1 Let $G$ be a finite group, for which every complex representation is equivalent to a real representation. Then for every $w \in G$

$$
\sum_{\chi \in G^{*}} \chi(w)=\#\left\{u \in G \mid u^{2}=w\right\}
$$

where $G^{*}$ denotes the set of the irreducible characters of $G$.

It is well known [22] that all complex representations of a Weyl group are equivalent to rational representations. In particular, Theorem 5.1 holds for $G=S_{n}$. One concludes

Corollary 5.2 Let $\pi \in S_{n}$ have cycle structure $1^{d_{1}} 2^{d_{2}} \cdots n^{d_{n}}$. Then

$$
\sum_{\chi \in S_{n} *} \chi(\pi)=\prod_{r=1}^{n} f\left(r, d_{r}\right),
$$

where

$$
f\left(r, d_{r}\right)= \begin{cases}0, & \text { if } r \text { is even and } d_{r} \text { is odd } \\
\left(\begin{array}{c}
d_{r} \\
2, \ldots, 2
\end{array}\right) \cdot r^{d_{r} / 2}, & \text { if } r \text { and } d_{r} \text { are even } \\
\sum_{k=0}^{\left\lfloor d_{r} / 2\right\rfloor}\left(\begin{array}{c}
d_{r} \\
d_{r}-2 k, 2,2, \ldots, 2
\end{array}\right) \cdot r^{k}, & \text { if } r \text { is odd } .\end{cases}
$$

In particular, $f(r, 0)=1$ for all $r$.

For a proof see [1].

\subsection{Proof of Theorem 2.2}

We shall compute the character of the representation $\rho$ and compare it with Corollary 5.2.

Recall the definition of the inversion set of a permutation $\pi \in S_{n}$,

$$
\operatorname{Inv}(\pi):=\{\{i, j\} \mid(j-i) \cdot(\pi(j)-\pi(i))<0\} .
$$

Definition 5.3 For an involution $w \in I_{n}$ let Pair $(w)$ be the set of 2-cycles of $w$ (considered as unordered 2-sets). For a permutation $\pi \in S_{n}$ and an involution $w \in I_{n}$ let

$$
\operatorname{Inv}_{w}(\pi):=\operatorname{Inv}(\pi) \cap \operatorname{Pair}(w)
$$

and

$$
\operatorname{inv}_{w}(\pi):=\# \operatorname{Inv}_{w}(\pi)
$$


Proposition 5.4 [1, equation (4)]

$$
\rho(\pi) C_{w}=(-1)^{\operatorname{inv}_{w}(\pi)} \cdot C_{\pi w \pi^{-1}} \quad\left(\forall \pi \in S_{n}, w \in I_{n}\right) .
$$

It follows that

$$
\operatorname{Tr}(\rho(\pi))=\sum_{w \in I_{n} \cap \mathrm{St}_{n}(\pi)}(-1)^{\operatorname{inv}_{w}(\pi)},
$$

where $\operatorname{St}_{n}(\pi)$ is the centralizer of $\pi$ in $S_{n}$ (i.e., the stabilizer of $\pi$ under conjugation).

Observation 5.5 Let $\pi \in S_{n}, w \in I_{n} \cap \mathrm{St}_{n}(\pi)$ and $a_{1} \in[n]$ any letter. Then one of the following holds:

(1) $\left(a_{1}, a_{2}, \ldots, a_{r}\right)$ is a cycle in $\pi(r \geq 1) ; a_{1}, a_{2}, \ldots, a_{r}$ are fixed points of $w$.

(2) $\left(a_{1}, a_{2}, \ldots, a_{r}\right)$ and $\left(a_{r+1}, \ldots, a_{2 r}\right)$ are cycles in $\pi(r \geq 1) ;\left(a_{1}, a_{r+1}\right),\left(a_{2}, a_{r+2}\right), \ldots,\left(a_{r}, a_{2 r}\right)$ are cycles in $w$.

(3) $\left(a_{1}, a_{2}, \ldots, a_{2 m}\right)$ is a cycle in $\pi(m \geq 1)$; $\left(a_{1}, a_{m+1}\right),\left(a_{2}, a_{m+2}\right), \ldots,\left(a_{m}, a_{2 m}\right)$ are cycles in w.

For every $A \subseteq[n]$ let $S_{A}:=\left\{\pi \in S_{n} \mid \operatorname{Supp}(\pi) \subseteq A\right\}$ be the subgroup of $S_{n}$ consisting of all permutations whose support is contained in $A$.

For every $\pi \in S_{n}$ and $1 \leq r \leq n$ let $A(\pi, r) \subseteq[n]$ be the set of all letters which appear in cycles of length $r$ in $\pi$. In other words,

$$
A(\pi, r):=\left\{i \in[n] \mid \pi^{r}(i)=i \text { and }(\forall j<r) \pi^{j}(i) \neq i\right\}
$$

For example, $A(\pi, 1)$ is the set of fixed points of $\pi$.

Denote by $\pi_{\mid r}$ the restriction of $\pi$ to $A(\pi, r)$. Then $\pi_{\mid r}$ may be considered as a permutation in $S_{A(\pi, r)}$. By Observation 5.5,

Corollary 5.6 Fix $\pi \in S_{n}$. Each $w \in I_{n} \cap \operatorname{St}_{n}(\pi)$ has a unique decomposition

$$
w=\prod_{r \geq 1} w_{r}
$$

where

$$
w_{r} \in I_{S_{A(\pi, r)}} \cap \operatorname{St}_{S_{A(\pi, r)}}\left(\pi_{\mid r}\right)
$$

with $A(\pi, r), \pi_{\mid r}$ and $S_{A(\pi, r)}$ as defined above; and

$$
\operatorname{Inv}_{w}(\pi)=\bigcup_{r \geq 1} \operatorname{Inv}_{w_{r}}\left(\pi_{\mid r}\right)
$$

a disjoint union.

Hence, it suffices to prove that $\operatorname{Tr}(\rho(\pi))$ is equal to the right hand side of the formula in Corollary 5.2, for $\pi$ of cycle type $r^{n / r}$. Since $\rho$ is a class function, we may assume that

$$
\pi=(1,2, \ldots, r)(r+1, \ldots, 2 r) \cdots(n-r+1, n-r+2, \ldots, n) .
$$


Observation 5.7 Let $r$ be a positive integer.

(1) If $i$ and $j$ are distinct nonnegative integers, $\pi$ as in (3) above, and $w=($ ir $+1, j r+\sigma(1))($ ir + $2, j r+\sigma(2)) \cdots(i r+r, j r+\sigma(r))$ (where $\sigma$ is some power of the cyclic permutatation $(1,2, \ldots, r)$ ), then

$$
(-1)^{\operatorname{inv}_{w}(\pi)}=1
$$

(2) If $r=2 m$ is even, $\pi$ as in (3) above, and $w=(1, m+1)(2, m+2) \cdots(m, 2 m)$, then

$$
(-1)^{\operatorname{inv}_{w}(\pi)}=-1
$$

Lemma 5.8 For every odd $r$ and a permutation $\pi$ as in (3) above,

$$
\sum_{w \in I_{n} \cap \mathrm{St}_{n}(\pi)}(-1)^{\operatorname{inv}_{w}(\pi)}=\#\left(I_{n} \cap \mathrm{St}_{n}(\pi)\right)=\sum_{k=0}^{\lfloor n / 2 r\rfloor}\left(\begin{array}{c}
n / r \\
n / r-2 k, 2,2, \ldots, 2
\end{array}\right) \cdot r^{k} .
$$

Proof of Lemma 5.8. If $r$ is odd then only cases (1) and (2) in Observation 5.5 are possible. The first equality in the statement of the lemma then follows from Observation 5.7(1). The second equality follows from Observation 5.5(1)(2), counting the involutions $w \in I_{n} \cap \operatorname{St}_{n}(\pi)$ with \# $\operatorname{Supp}(w)=2 r k$.

Lemma 5.9 For every even $r$ and a permutation $\pi$ as in (3) above,

$$
\sum_{w \in I_{n} \cap \mathrm{St}_{n}(\pi)}(-1)^{\operatorname{inv}_{w}(\pi)}= \begin{cases}0, & \text { if } n / r \text { is odd } \\
\left(\begin{array}{c}
n / r \\
2, \ldots, 2
\end{array}\right) \cdot r^{n / 2 r}, & \text { if } n / r \text { is even }\end{cases}
$$

Proof of Lemma 5.9. Let $c_{i}=(i r+1, i r+2, \ldots, i r+r)$ be one of the cycles of $\pi$, as in (3). By Observation 5.5, an involution $w \in I_{n} \cap \mathrm{St}_{n}(\pi)$ has one of the following three types with respect to $c_{i}$ :

Type (1): Each element of $c_{i}$ is a fixed point of $w$.

Type (2): $w$ maps $c_{i}$ onto a different cycle of $\pi$.

Type (3): $r=2 m$ is even, and $c_{i}$ is a union of 2-cycles of $w$ :

$$
\{i r+t, i r+t+m\} \in \operatorname{Pair}(w) \quad(1 \leq t \leq m) .
$$

Denote

$$
P_{2}:=\left\{w \in I_{n} \cap \mathrm{St}_{n}(\pi) \mid w \text { is of type (2) w.r.t. all cycles of } \pi\right\} .
$$

For any $w \in\left(I_{n} \cap \operatorname{St}_{n}(\pi)\right) \backslash P_{2}$, let

$$
i(w):=\min \left\{i \mid w \text { is of type (1) or (3) w.r.t. the cycle } c_{i}\right\} .
$$

Denote

$$
P_{1}:=\left\{w \in\left(I_{n} \cap \mathrm{St}_{n}(\pi)\right) \backslash P_{2} \mid w \text { is of type (1) w.r.t. the cycle } c_{i(w)}\right\}
$$


and

$$
P_{3}:=\left\{w \in\left(I_{n} \cap \operatorname{St}_{n}(\pi)\right) \backslash P_{2} \mid w \text { is of type (3) w.r.t. the cycle } c_{i(w)}\right\} .
$$

The map $\varphi: P_{1} \rightarrow P_{3}$ which changes the action of $w$ on $c_{i(w)}$ from type (1) to type (3) is clearly a well-defined bijection; and, by Observation 5.7(2), it reverses the sign of $(-1)^{\operatorname{inv}_{w}(\pi)}$. The contributions of $P_{1}$ and $P_{3}$ to the sum therefore cancel each other. Each element of the remaining set $P_{2}$ contributes 1 , by Observation 5.7(1). Lemma 5.9 follows.

Lemmas 5.8 and 5.9 complete the proof of Theorem 2.2.

\section{The Involutive Length}

Recall the definition of the involutive length $\hat{\ell}$ (Definition 3.1). In order to prove Theorem 3.2 we need the following combinatorial interpretation of the involutive length $\hat{\ell}$.

Lemma 6.1 Let $w \in S_{n}$ be an involution of cycle type $2^{k} 1^{n-2 k}$. Then

$$
\hat{\ell}(w):=\left[\sum_{t \in \operatorname{Supp}(w)} t-\left(\begin{array}{c}
2 k+1 \\
2
\end{array}\right)\right]+\frac{1}{2}\left[\operatorname{inv}\left(w_{\mid \operatorname{Supp}(w)}\right)-k\right] .
$$

Proof: Denote the right hand side of (4) by $f(w)$. It is easy to verify that $f(w)=0$ when $\hat{\ell}(w)=0$, i.e., when $w=s_{1} s_{3} \cdots s_{2 k-1}$. Let $u$ and $v=s_{i} u s_{i}$ be involutions in $S_{n}$ with $\hat{\ell}(v)=\hat{\ell}(u)+1$. Then $|\{i, i+1\} \cap \operatorname{Supp}(u)|>0$. If $|\{i, i+1\} \cap \operatorname{Supp}(u)|=1$ then

$$
\sum_{t \in \operatorname{Supp}(v)} t-\sum_{t \in \operatorname{Supp}(u)} t= \pm 1
$$

and $\operatorname{inv}\left(v_{\mid \operatorname{Supp}(v)}\right)=\operatorname{inv}\left(u_{\mid \operatorname{Supp}(u)}\right)$. If $|\{i, i+1\} \cap \operatorname{Supp}(u)|=2$ then

$$
\sum_{t \in \operatorname{Supp}(v)} t=\sum_{t \in \operatorname{Supp}(u)} t
$$

and $\operatorname{inv}\left(v_{\mid \operatorname{Supp}(v)}\right)-\operatorname{inv}\left(u_{\mid \operatorname{Supp}(u)}\right) \in\{2,0,-2\}$. Thus in both cases $|f(v)-f(u)| \leq 1$. This proves, by induction on $\hat{\ell}$, that $f(w) \leq \hat{\ell}(w)$ for every involution $w$.

On the other hand, if $w$ is an involution with $f(w)>0$ then either $\sum_{t \in \operatorname{Supp}(w)} t>\left(\begin{array}{c}2 k+1 \\ 2\end{array}\right)$, or $\sum_{t \in \operatorname{Supp}(w)} t=\left(\begin{array}{c}2 k+1 \\ 2\end{array}\right)$ and $\operatorname{inv}\left(w_{\mid \operatorname{Supp}(w)}\right)>k$. In the first case there exists $i+1 \in \operatorname{Supp}(w)$ such that $i \notin \operatorname{Supp}(w)$. Then $f\left(s_{i} w s_{i}\right)=f(w)-1$. In the second case $\operatorname{Supp}(w)=\{1, \ldots, 2 k\}$. Since $\operatorname{inv}\left(w_{\mid \operatorname{Supp}(w)}\right)>k, w \neq s_{1} s_{3} \cdots s_{2 k-1}$. Thus there must exist a minimal $i$ satisfying $w(i)>i+1$. Denoting $j:=w(i)-1>i$ we cannot have $w(j)<i$ (by minimality of $i$ ), and therefore $w(j)>i=w(j+1)$ and $f\left(s_{j} w s_{j}\right)=f(w)-1$. We conclude that $\hat{\ell}(w) \leq f(w)$ for every involution $w$. 


\section{The Hecke Algebra}

\subsection{Proof of Theorem 3.2(1).}

We prove that $\rho_{q}$ is an $H_{n}(q)$-representation by verifying the defining relations.

First, consider the braid relation $T_{i} T_{i+1} T_{i}=T_{i+1} T_{i} T_{i+1}$. To verify this relation observe that there are six possible types of orbits of an involution $w$ under conjugation by $\left\langle s_{i}, s_{i+1}\right\rangle$ - the subgroup in $S_{n}$ generated by $s_{i}$ and $s_{i+1}$. These orbits differ by the action of $w$ on the letters $i, i+1, i+2$ :

1. $i, i+1, i+2 \notin \operatorname{Supp}(w)$.

2. Exactly one of the letters $i, i+1, i+2$ is $\operatorname{in} \operatorname{Supp}(w)$.

3. Exactly two of the letters $i, i+1, i+2$ are in $\operatorname{Supp}(w)$, and these two letters form a 2-cycle in $w$.

4. Exactly two of the letters $i, i+1, i+2$ are in $\operatorname{Supp}(w)$, and these two letters do not form a 2-cycle in $w$.

5. $i, i+1, i+2 \in \operatorname{Supp}(w)$, and two of these letters form a 2-cycle in $w$.

6. $i, i+1, i+2 \in \operatorname{Supp}(w)$, and no two of these letters form a 2-cycle in $w$.

Note that an orbit of the first type is of order one; orbits of the second, third and fifth type are of order three; and orbits of the fourth and sixth type are of order six. Moreover, by Lemma 6.1, orbits of the same order form isomorphic intervals in the weak involutive order (see Definition 3.1). In particular, all orbits of order six have a representative $w$ of minimal involutive length, such that the orbit has the form :

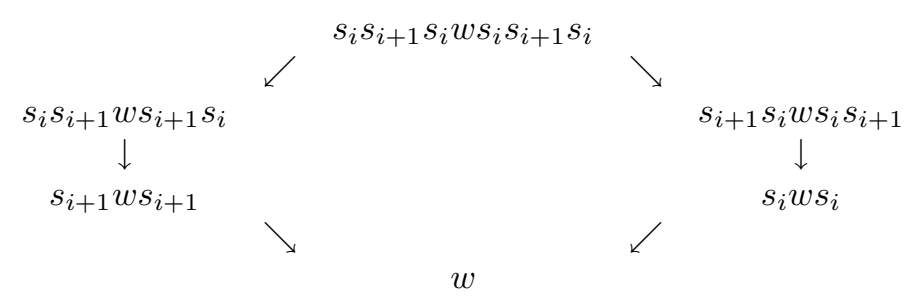

$w$

All orbits of order three are linear posets:

$$
w \prec_{I} s_{i} w s_{i} \prec_{I} s_{i+1} s_{i} w s_{i} s_{i+1},
$$

or

$$
w \prec_{I} s_{i+1} w s_{i+1} \prec_{I} s_{i} s_{i+1} w s_{i+1} s_{i} .
$$

Thus the analysis is reduced into three cases.

Case (a). An orbit of order six. By (2) and (5), the representation matrices of the generators with respect to the ordered basis $C_{w}, C_{s_{i} w s_{i}}, C_{s_{i+1} s_{i} w s_{i} s_{i+1}}, C_{s_{i} s_{i+1} s_{i} w s_{i} s_{i+1} s_{i}}, C_{s_{i+1} w s_{i+1}}, C_{s_{i} s_{i+1} w s_{i+1} s_{i}}$ are :

$$
\rho_{q}\left(T_{i}\right)=\left(\begin{array}{cccccc}
1-q & 1 & 0 & 0 & 0 & 0 \\
q & 0 & 0 & 0 & 0 & 0 \\
0 & 0 & 1-q & 1 & 0 & 0 \\
0 & 0 & q & 0 & 0 & 0 \\
0 & 0 & 0 & 0 & 1-q & 1 \\
0 & 0 & 0 & 0 & q & 0
\end{array}\right)
$$


and

$$
\rho_{q}\left(T_{i+1}\right)=\left(\begin{array}{cccccc}
1-q & 0 & 0 & 0 & 1 & 0 \\
0 & 1-q & 1 & 0 & 0 & 0 \\
0 & q & 0 & 0 & 0 & 0 \\
0 & 0 & 0 & 0 & 0 & q \\
q & 0 & 0 & 0 & 0 & 0 \\
0 & 0 & 0 & 1 & 0 & 1-q
\end{array}\right)
$$

It is easy to verify that indeed

$$
\rho_{q}\left(T_{i}\right) \rho_{q}\left(T_{i+1}\right) \rho_{q}\left(T_{i}\right)=\rho_{q}\left(T_{i+1}\right) \rho_{q}\left(T_{i}\right) \rho_{q}\left(T_{i+1}\right)
$$

Case (b). An orbit of order three. With no loss of generality, the orbit is of type (6); the analysis of type (7) is analogous. Then $s_{i+1} w s_{i+1}=w$ and $s_{i}\left(s_{i+1} s_{i} w s_{i} s_{i+1}\right) s_{i}=s_{i+1} s_{i} w s_{i} s_{i+1}$. It is shown in [1] that $s_{i+1} \in \operatorname{Des}(w)$ if and only if $s_{i} \in \operatorname{Des}\left(s_{i+1} s_{i} w s_{i} s_{i+1}\right)$.

Given the above, by (2), the representation matrices of the generators with respect to the ordered basis $w \prec_{I} s_{i} w s_{i} \prec_{I} s_{i+1} s_{i} w s_{i} s_{i+1}$ are

$$
\rho_{q}\left(T_{i}\right)=\left(\begin{array}{ccc}
1-q & 1 & 0 \\
q & 0 & 0 \\
0 & 0 & x
\end{array}\right)
$$

and

$$
\rho_{q}\left(T_{i+1}\right)=\left(\begin{array}{ccc}
x & 0 & 0 \\
0 & 1-q & 1 \\
0 & q & 0
\end{array}\right),
$$

where $x \in\{1,-q\}$. These matrices satisfy the braid relation.

Case (c). An orbit of order one. Then $s_{i} w s_{i}=w, s_{i+1} w s_{i+1}=w$ and $s_{i}, s_{i+1} \notin \operatorname{Des}(w)$. By (2), $\rho_{q}\left(T_{i}\right) \rho_{q}\left(T_{i+1}\right) \rho_{q}\left(T_{i}\right) C_{w}=\rho_{q}\left(T_{i+1}\right) \rho_{q}\left(T_{i}\right) \rho_{q}\left(T_{i+1}\right) C_{w}=C_{w}$, completing the proof of the third relation.

The proof of the other two relations is easier and will be left to the reader.

Remark 7.1 Substituting $q=1$ proves Theorem 2.1 .

\subsection{Proof of Theorem 3.2(2).}

We apply Lusztig's version of Tits' deformation theorem to prove that $\rho_{q}$ is a Gelfand model.

Consider the Hecke algebra $H_{n}(q)$ as the algebra over $\mathbb{Q}\left(q^{1 / 2}\right)$ spanned by $\left\{T_{v} \mid v \in S_{n}\right\}$ with the multiplication rules

$$
T_{v} T_{u}=T_{v u} \quad \text { if } \ell(v u)=\ell(v)+\ell(u)
$$

and

$$
\left(T_{s}+q\right)\left(T_{s}-1\right)=0 \quad(\forall s \in S)
$$


By Lusztig's version of Tits' deformation theorem [17, Theorem 3.1], the group algebra of $S_{n}$ over $\mathbb{Q}\left(q^{1 / 2}\right)$ may be embedded in $H_{n}(q)$. In particular, every element $w \in S_{n}$ may be expressed as a linear combination

$$
w=\sum_{v \in S_{n}} m_{v, w}\left(q^{1 / 2}\right) T_{v},
$$

where $m_{v, w}$ is a rational function of $q^{1 / 2}$.

It follows that $\rho_{q}$ may be considered as an $S_{n}$-representation, via

$$
\rho_{q}(w):=\sum_{v \in S_{n}} m_{v, w}\left(q^{1 / 2}\right) \rho_{q}\left(T_{v}\right) \quad\left(\forall w \in S_{n}\right) .
$$

The resulting character values $\rho_{q}(w)$ are rational functions of $q^{1 / 2}$. By discreteness of the $S_{n}$ character values, each such function is locally constant wherever it is defined, and is thus constant globally. each such fu By Theorem 2.2, $\left.\rho_{q}\right|_{q=1}=\rho$ is a model for the group algebra of $S_{n}$. This completes the proof.

\subsection{Proof of Proposition 3.3}

Let $\mathrm{SYT}_{n}$ be the set of all standard Young tableaux of order $n$, and let $\operatorname{SYT}(\lambda) \subseteq \mathrm{SYT}_{n}$ be the set of all standard Young tableaux of shape $\lambda$. For each partition $\lambda$ of $n$, fix a standard Young tableau $P_{\lambda} \in \operatorname{SYT}(\lambda)$. By [20, Theorem 4], the value of the irreducible $H_{n}(q)$-character $\chi_{q}^{\lambda}$ at $T_{\mu}$ is

$$
\chi_{q}^{\lambda}\left(T_{\mu}\right)=\sum_{\left\{w \mapsto\left(P_{\lambda}, Q\right) \mid w \text { is } \mu \text {-unimodal and } Q \in \operatorname{SYT}(\lambda)\right\}}(-q)^{\operatorname{des}(w)},
$$

where the sum runs over all $\mu$-unimodal permutations $w \in S_{n}$ which are mapped under the RobinsonSchensted (RS) correspondence to $\left(P_{\lambda}, Q\right)$ for some $Q \in \operatorname{SYT}(\lambda)$. By [23, Lemma 7.23.1], the descent set of such $w \in S_{n}$ is determined by $Q$. Hence

$$
\begin{gathered}
\left.\operatorname{Tr} \rho_{q}\left(T_{\mu}\right)=\sum_{\lambda} \chi_{q}^{\lambda}\left(T_{\mu}\right)\right)=\sum_{\lambda} \sum_{\left\{w \mapsto\left(P_{\lambda}, Q\right) \mid w \text { is } \mu \text {-unimodal and } Q \in \operatorname{SYT}(\lambda)\right\}}(-q)^{\operatorname{des}(w)} \\
=\sum_{\lambda} \sum_{\{w \mapsto(Q, Q) \mid w \text { is } \mu \text {-unimodal and } Q \in \operatorname{SYT}(\lambda)\}}(-q)^{\operatorname{des}(w)} \\
=\sum_{\left\{w \mapsto(Q, Q) \mid Q \in \operatorname{SYT}_{n} \text { and } w \text { is } \mu \text {-unimodal }\right\}}(-q)^{\operatorname{des}(w)}=\sum_{\left\{w \in I_{n} \mid w \text { is } \mu \text {-unimodal }\right\}}(-q)^{\operatorname{des}(w)} .
\end{gathered}
$$

The last equality follows from the well known property of the RS correspondence: $w \mapsto(P, Q)$ if and only if $w^{-1} \mapsto(Q, P)$ [23, Theorem 7.13.1]. Thus $w$ is an involution if and only if $w \mapsto(Q, Q)$ for some $Q \in \mathrm{SYT}_{n}$. 


\section{Remarks and Questions}

Models for classical Weyl groups of type $D_{n}$ for odd $n$ were constructed in $[6,5]$. These constructions fail for even $n$. A natural question is whether there exists a signed conjugation (or a representation of type $\rho_{s} C_{w}=a_{s, w} C_{w}+b_{s, w} C_{s w s}$ ) which gives a model for $D_{2 n}$. It is also desired to find representation matrices for the models of the Hecke algebras of types $B$ and $D$ which specialize at $q=1$ to models of the corresponding group algebra.

We conclude with the following questions regarding an arbitrary Coxeter group $W$.

Question 8.1 Find a signed conjugation which gives a Gelfand model for $W$; Find a representation of the form $\rho_{s} C_{w}=a_{s, w} C_{w}+b_{s, w} C_{s w s}$, which gives a Gelfand model for the Hecke algebra of $W$.

Question 8.2 Find a character formula for the Gelfand model of the Hecke algebra of $W$.

\section{Added in Proof}

First, it should be acknowledged that an equivalent reformulation of Theorem 2.2, with a different proof, was given by Kodiyalam and Verma [15].

A third proof of Theorem 2.2, along the lines of [11], was suggested by an anonymous referee of [1]. Here is a brief outline.

Let $\chi^{\emptyset,(n)}$ denote the one dimensional character of $B_{n}$ given by the parity of the number of negative signs, and consider the natural embedding of $B_{n}=\mathbb{Z}_{2} 2 S_{n}$ into $S_{2 n}$. Combining [18, Ch. I $\S 8$ Ex 6, and Ch. VII (2.4)] with the Littlewood-Richardson rule implies that

$$
\left(\left(\chi^{\emptyset,(k)} \uparrow_{B_{k}}^{S_{2 k}}\right) \otimes 1_{S_{n-2 k}}\right) \uparrow_{S_{2 k} \times S_{n-2 k}}^{S_{n}}
$$

is a multiplicity free sum of all irreducible Specht modules indexed by partitions with exactly $n-2 k$ odd columns. A natural basis for this representation is given by involutions with $n-2 k$ fixed points. Finally, it is straightforward to show that the action of a Coxeter generator $s_{i}$ on this basis is identical with the signed conjugation defined in (1).

Corollary 9.1 The signed conjugation $\rho$, when restricted to the conjugacy classes of involution with $n-2 k$ fixed points, is a multiplicity free sum of all irreducible Specht modules indexed by partitions with exactly $n-2 k$ odd columns.

This gives an algebraic proof to the following enumerative result: The number of involutions with $n-2 k$ fixed points is equal to the number of standard Young tableaux of shapes with exactly $n-2 k$ odd columns. Another proof for this fact may be obtained using the Robinson-Schensted correspondence. One concludes that $S^{\lambda}$ is a factor of the restriction of $\rho$ to a conjugacy class of cycle type $2^{k} 1^{n-2 k}$ if and only if $\lambda$ is the shape of some pair of (equal) standard Young tableaux corresponding to an involution of this cycle type. 


\section{Acknowledgements}

The authors thank Arkady Berenstein, Steve Shnider and Richard Stanley for stimulating discussions and references. We also thank the anonymous referees for helpful suggestions.

\section{References}

[1] R. M. Adin, A. Postnikov and Y. Roichman, Combinatorial Gelfand models. J. Algebra, to appear.

[2] R. M. Adin, A. Postnikov and Y. Roichman, A Gelfand model for wreath products. preprint, 2008.

[3] J. L. Aguado and J. O. Araujo, A Gelfand Model for the Symmetric Group. Communications in Algebra 29 (2001), 1841-1851

[4] J. O. Araujo, A Gelfand model for a Weyl group of type $B_{n}$. Beiträge Algebra Geom. 44 (2003), 359-373.

[5] J. O. Araujo, and J. J. Bigeón, A Gelfand model for a Weyl group of type $D_{n}$ and the branching rules $D_{n} \hookrightarrow B_{n}$. J. Algebra 294 (2005), 97-116.

[6] R. W. Baddeley, Models and involution models for wreath products and certain Weyl groups. J. London Math. Soc. (2) 44 (1991), 55-74.

[7] C. T. Benson and C. W. Curtis, On the degrees and rationality of certain characters of finite Chevalley groups. Trans. Amer. Math. Soc. 165 (1972), 251-273; 202 (1975) 405-405.

[8] I. N. Bernstein, I. M. Gelfand and S. I. Gelfand, Models of representations of compact Lie groups. (Russian) Funksional. Anal. i Prilozen. 9 (1975), 61-62.

[9] N. Bourbaki, Lie Groups and Lie Algebras. English translation by Andrew Pressley, Springer, 2002.

[10] C. W. Curtis and I. Reiner, Methods of Representation Theory, Vol. II. With applications to finite groups and orders. Pure and Applied Mathematics (New York), John Wiley \& Sons, Inc., New York, 1987

[11] N. F. J. Inglis, R. W. Richardson, J. Saxl, An explicit model for the complex representations of $S_{n}$. Arch. Math. (Basel) 54 (1990), 258-259.

[12] I. M. Isaacs, Character Theory of Finite Groups. Dover, New York, 1994.

[13] A. A. Klyachko, Models for complex representations of the groups $\operatorname{GL}(n, q)$ and Weyl groups. (Russian) Dokl. Akad. Nauk SSSR 261 (1981), 275-278.

[14] A. A. Klyachko, Models for complex representations of groups $\operatorname{GL}(n, q)$. (Russian) Mat. Sb. (N.S.) 120(162) (1983), 371-386.

[15] V. Kodiyalam and D.-N. Verma, A natural representation model for symmetric groups. preprint, 2004. 
[16] G. Kudryavtseva and V. Mazorchuk, Combinatorial Gelfand models for some semigroups and q-rook monoid algebras. preprint, 2007.

[17] G. Lusztig, On a theorem of Benson and Curtis. J. Algebra 71 (1981), 490-498.

[18] I. G. Macdonald, Symmetric Functions and Hall Polynomials. second edition, Oxford Math. Monographs, Oxford Univ. Press, Oxford, 1995.

[19] A. Melnikov, B-orbits of nilpotent order 2 and link patterns. preprint, 2007.

[20] Y. Roichman, A recursive rule for Kazhdan-Lusztig characters. Adv. in Math. 129 (1997), 24-45.

[21] P. D. Ryan, Representations of Weyl groups of type B induced from centralisers of involutions. Bull. Austral. Math. Soc. 44 (1991), 337-344.

[22] T. A. Springer, A construction of representations of Weyl groups. Invent. Math. 44 (1978), 279-293.

[23] R. P. Stanley, Enumerative Combinatorics, Volume II. Cambridge Univ. Press, Cambridge, 1999. 
